\title{
Fortgeschrittene biomolekülbasierte Architekturen auf Elektroden für die Entwicklung neuartiger Biosensoren
}

\author{
S. C. Feifel", F. Lisdat \\ Institut für Angewandte Biowissenschaften, Technische Hochschule Wildau, \\ Hochschulring 1, 15745 Wildau, Deutschland \\ *feifel@th-wildau.de
}

\begin{abstract}
Kurzfassung:
Aus drei unterschiedlichen Proteinkomponenten wurden vier supramolekulare Proteinarchitekturen auf Elektroden für den selektiven Substratnachweis aufgebaut. Der supramolekulare Aufbau im Multischichtformat wird durch das Einbetten eines Redoxproteins (Cytochrom c) sowie zweier unterschiedlicher Enzyme (Cellobiosedehydrogenase und Laccase) mittels carboxymodifizierter Siliziumdioxidnanopartikeln realisiert. Die unterschiedlichen Multiproteinsysteme ermöglichen jeweils die Einzeldetektion als auch in dem Triproteinsystem eine duale Detektion zweier unterschiedlicher Analyte: Laktose und Sauerstoff. Die Kontrolle der Enzymaktivität - das heißt, das selektive Anschalten der Bioelektrokatalyse - wird durch das angelegte Potential erreicht, da hierdurch der Redoxzustand von Cytochrom $c$ vorgegeben wird. Die beiden bidirektionalen Signalketten im dem Triproteinansatz arbeiten in einer unseparierten Matrix, ohne dass die Anwesenheit des anderen Biokatalysators störend wirkt. Damit konnte eine bidirektionale Elektronentransferkette basierend auf intermolekularen Elektronentransferschritten zwischen immobilisierten Biomolekülen etabliert werden.
\end{abstract}

Schlagwörter: Biosensor, Nanopartikel, Multischichten, Enzyme, Cytochrom c.

\section{Einleitung}

Seit den ersten Konzepten in den 1960zigern, erfolgte eine stetige Weiterentwicklung der Biosensoren. Innerhalb der verschiedenen Arten von Biosensoren stellen die elektrochemischen Sensoren noch immer den größten Anteil [1]. Die Immobilisierung der Biokomponente, welchen einen Analyten spezifisch erkennt, ist ein entscheidender Schritt bei der Konstruktion eines Biosensors [2]. Sehr oft kommt es zu einer reduzierten Aktivität der Biomoleküle an den Grenzflächen (Fest-Flüssig), was eine adäquate Anpassung der Oberfläche und Fixierung des Biomoleküls erfordert [3]. Zudem ist die Menge an Biomolekül, die immobilisiert werden kann, entscheidend für die Empfindlichkeit des Sensors. Dies führte unter anderem zur Entwicklung von Multischichtbasierten Immobilisierungsverfahren (Layer-by-Layer Prinzip) in denen ein Biomolekül oder unterschiedliche Arten von Biomolekülen in Kombination mit einer Matrix schichtartig assembliert wurden [4]. Für die Einbettung von Enzymen in einer Multischichtarchitektur spielt die elektrische Kommunikation und die damit einhergehende Elektronentransferreaktionen (ET) der Moleküle mit der Elektrode eine entscheidende Rolle und ist von grundlegender Bedeutung für die Ent- wicklung enzym-basierter Biosensorsysteme [57]. Hierbei gibt es verschiedene Grundprinzipien - über den Nachweis von Reaktionsprodukten der Enzymreaktion und Mediatorbasiertem Elektronentransfer bis hin zu einer direkten elektrochemischen Umwandlung von Redoxenzymen. Letztere Systeme, die ohne Shuttlemoleküle auskommen, sind im Fokus unserer Untersuchungen. Die Geschwindigkeit und Effizienz des Elektronentransfers zwischen einem Enzym und einer Elektrode ist abhängig von der Lage des Redox-Zentrums des Enzyms, der molekularen Umgebung des Redoxzentrums, und der Orientierung des Enzyms auf der Elektrodenoberfläche und innerhalb der Multischicht $[8,9]$. Basierend auf diesen Annahmen ist es notwendig eine Architektur aufzubauen, in welcher die eingebetteten Enzyme und Redoxproteine in der Lage sind effizient Elektronen miteinander auszutauschen. Für den Aufbau von multischicht-basierten enzymatischen Biosensoren ist die Auswahl eines geeigneten Matrixmoleküls auch von entscheidender Bedeutung, da es die Funktionalität des Biomoleküls nicht negativ beeinträchtigen solIte, aber auch eine ausreichende Interaktion mit dem Biomolekül gewährleistet sein muss um es für den modularen Schichtaufbau einzusetzen. Siliziumdioxidnanopartikel (SiNPs) wurden unter diesem Aspekt schon für den Aufbau von 
Biosensoren aufgrund ihrer guten Biokompatibilität und guten Stabilität erfolgreich genutzt [10-12].

Basierend auf diesen Grundlagen, entwickelten wir unter Nutzung von SiNPs, Cytochrom $c$ (Cyt c), Laccase (Lac) und Cellobiosedehydrogenase $(\mathrm{CDH})$ neue funktionelle supramolekulare Biosensorarchitekturen auf Elektroden für den spezifischen Analytnachweis.

\section{Ergebnisse und Diskussion}

Für den Aufbau der unterschiedlichen enzymbasierten Multischicht-Systeme wurden Siliziumdioxidnanopartikel (SiNPs) als Matrixelemente verwendet. Um eine elektrostatische Wechselwirkung zwischen den Proteinen und den nichtleitenden SiNPs für den schichtweisen Aufbau zu gewährleisten, wurden diese mit Carboxyfunktionen versehen. Einen weiteren zentralen Baustein bildet das Redoxprotein Cyt $c$ welches als Elektronenshuttle zwischen den eingesetzten Enzymen und der Elektrode fungieren soll. Um die Funktionalität des Redoxproteins in einem SiNPs-basierten Multischichtsystem zu gewährleisten wurde dieses als erstes auf seine Funktionalität hin untersucht. Dafür wurden multiple Schichten von Cyt $c$ und SiNPs auf einer Cyt $c$-Monoschichtelektrode mithilfe alternierender Inkubationsschritte in den entsprechenden Lösungen, aus SiNPs, sowie Cyt $c$ aufgebaut. Die dem System zugrundeliegende Monoschichtelektrode besteht aus einer Goldelektrode, die mit einer gemischten Thiolschicht (Mercaptoundecansäure (MUA) und Mercaptoethanol (MU)) modifiziert wurde und an die Cyt $c$ adsorbiert ist. Die Cyt $c$-basierte Multischichtelektrode ist in Abbildung 1 schematisch dargestellt.

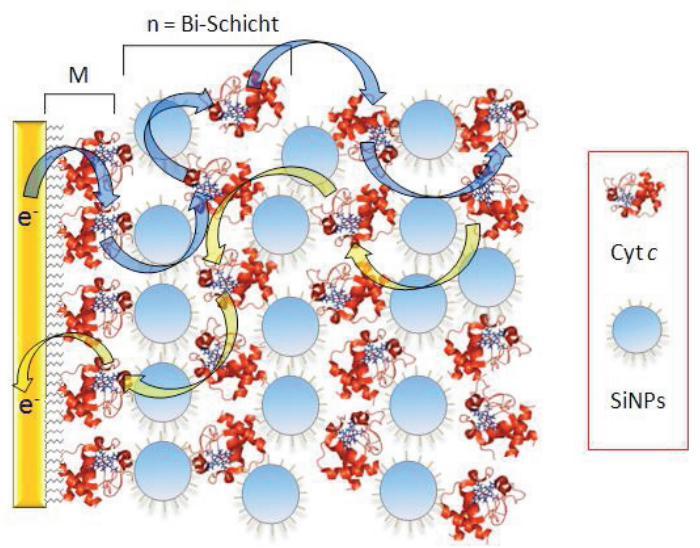

Abb. 1. Schematische

Darstellung

einer SiNPs/Cyt c Multischicht auf einer Monoschichtelektrode (M). Schichtstruktur [SiNPs/cyt c $]_{n}(n=1,2$, 3, 4-Bi-Schichten). Die gelben und blauen Pfeile zeigen den Elektronentransfer zwischen den Cytc Molekülen in der Architektur sowie den Transfer hinund weg von der Elektrode.
Die elektrochemischen Eigenschaften der Cyt $c / \mathrm{SiNPs}_{1-4}$ Multischichtelektroden wurden anschließend mittels zyklovoltammetrischen Messungen (CV) untersucht. Dafür wurden die Proteinarchitekturen bei höheren Scanraten charakterisiert. Hierbei zeigt sich, dass die Cyt $c$-Moleküle innerhalb der Schichtarchitektur elektroaktiv vorliegen (Abbildung 2). Die Menge an adressierbarem Cyt $c$ korreliert direkt mit der Zahl an Aufbauschritten (vier Multischichten entsprechen ca. $\left.90 \mathrm{pmol} / \mathrm{cm}^{2}\right)$. Die deutliche Zunahme an elektroaktivem Cyt $c$ beweist den effektiven Cyt $c$-Cyt $c$-Elektronenaustausch innerhalb des multiplen Schichtsystems. Zugleich kann gezeigt werden, dass die SiNPs eine funktionelle Matrix für den definierten Multischichtaufbau darstellen. Mit jedem Depositionsschritt kann eine ähnliche Menge an elektroaktivem Cyt $c$ immobilisiert werden.

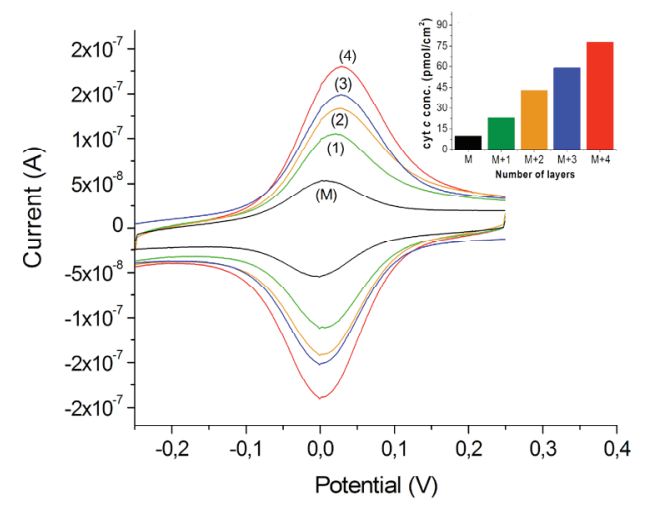

Abb. 2. Zyklovoltammogramm von Cyt c/SiNPs-basierten Multischichten. Zyklovoltammogramm vom 1, 2, 3, 4-Bi-Schichten; Cyt c/SiNPs $(\varnothing=5 \mathrm{~nm})$ und einer Cyt c-Monoschicht (M: Au-MU/MUA-Cyt c) für den direkten Vergleich. Inset: Auswertung der elektrochemisch bestimmten Cyt c-Konzentrationen in den einzelnen Multischichten [1, 2, 3, 4-BiSchichten], im Vergleich zu einer Monoschicht (M). Scanrate $100 \mathrm{mV} / \mathrm{s}$, Kalium-Phosphat Puffer $5 \mathrm{mM}$, pH 7.

Die Kommunikation der Cyt $c$-Moleküle in den einzelnen Proteinstapeln bildet jedoch nur eine Grundvoraussetzung für ein funktionsfähiges System in welches weitere Enzyme als Biokatalysator eingebettet werden sollen um einen Substratnachweis zu realisieren. Als erstes wurden neue, Cyt $c$-basierte Multischichtarchitekturen unter Verwendung von Cellobiosedehydrogenase $(\mathrm{CDH})$ für den selektiven Substratnachweis von Laktose aufgebaut. Um eine definierte Anordnung der Proteine zu erreichen, machten wir uns die unterschiedlichen Ladungen der Proteine zu Nutze. Da die CDH bei $\mathrm{pH} 7$ eine negative Nettoladung aufweist und Cyt $c$ eine positive, ist es möglich diese für die Bildung von Präkomplexen in einer definierten Mischung beider Proteine zu nutzen. Für eine ausreichend starke Wechselwirkung mit den 
negativ geladenen SiNPs ist jedoch ein Überschuss an Cyt $c$ erforderlich. Die Multischichtformierung mit Cyt $c, \mathrm{CDH}$ und SiNPs kann mittels Quarzkristallmikrowaage-(QCM)-Experimenten auf einer Goldoberfläche bestätigt werden. Anhand der QCM-Experimente kann eine definierte Bindung der Komponenten an die Oberfläche und der Komponenten untereinander gezeigt werden. Per Definition wird das Schichtsystem, das in zwei Immobilisierungsschritten erhalten wird (SiNPs, CDH/ Cyt c), als Zweischichtarchitektur (Bi-Schicht) bezeichnet. Die resultierende Struktur ist in Abb. 3 dargestellt.

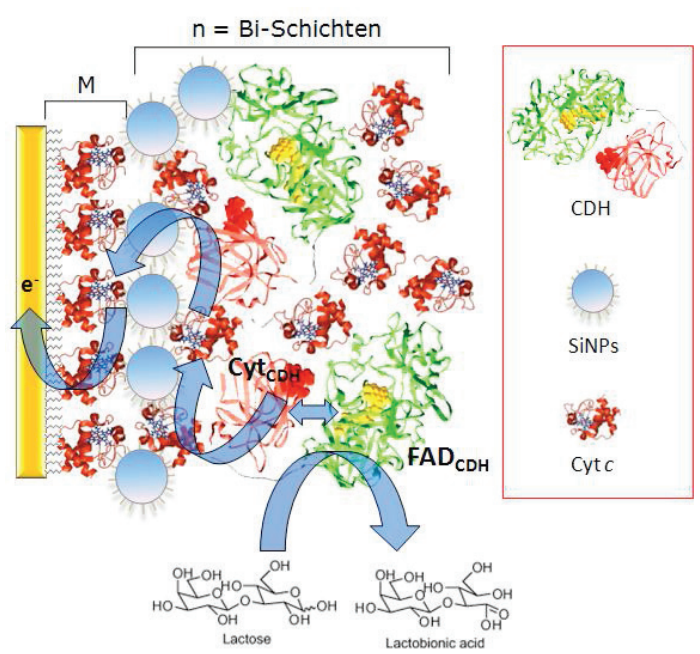

Fig. 3. Schematische Darstellung einer Supramolekularen [SiNP/CDH・Cyt c] Architektur, assembliert auf einer Cyt c-Monoschichtelektrode (M). Die Cyt c Monoschicht wurde auf einer gemischten Thiolschicht (MU/MUA) aufgebaut. Die Schichtstruktur ist [SiNPs/CDH•cyt c] $n$ ( $n=1,2,3,4-B i-S c h i c h t e n)$.

Um die katalytischen Eigenschaften der $\mathrm{CDH}$ innerhalb des modularen Aufbaus zu untersuchen, wurden CV-Messungen bei kleinen Scanraten $(3 \mathrm{mV} / \mathrm{s})$ in laktosefreiem bzw. laktosehaltigem Puffer durchgeführt. Hierbei kann in Gegenwart des Substrats ein deutlicher Oxidationsstrom beginnend ab einem Potential von -0.05 V gegen $\mathrm{Ag} / \mathrm{AgCl}$ (Abb. 4) beobachtet werden. Der katalytische Oxidationsstrom resultiert aus der Laktoseumsetzung durch die CDH-Moleküle. Da die Katalyse nur im Potentialbereich der Cyt $c$-Umwandlung auftritt, kann die Reaktionskaskade wie folgt beschrieben werden: Zuerst erfolgt die katalytische Oxidation der Laktose an der $\mathrm{CDH}$, die hierdurch aufgenommenen Elektronen werden wiederum an Cyt $c$ weitergegeben und mittels Interproteinelektronenransfer zwischen den Cyt cMolekülen zur Elektrode transportiert. Zusätzlich zeigen Kontrollexperimente, dass im Fall, wenn ausschließlich Cyt $c$ immobilisiert wurde, kein katalytischer Strom durch die Zugabe von Laktose beobachtet werden kann.
Weiterführend wurde die Effizienz der Signalkette beurteilt. Dafür wurde die katalytische Reaktion in Abhängigkeit der Zahl an immobilisierten Schichten untersucht. Zu diesem Zweck wurden Elektroden mit einer unterschiedlichen Zahl an [Cyt $c /$ SiNPs/Cyt $c \cdot C D H]_{n^{-}}$ Bi-Schichten aufgebaut und mittels $\mathrm{CV}$ bei $\mathrm{pH}$ 4.5 untersucht (Abb. 4). Anhand der CV-Daten kann gezeigt werden, dass sich mit zunehmender Zahl an Bi-Schichten auch der katalytische Strom nahezu linear erhöht. Für BiSchichtelektroden mit $n=4$ ist eine siebenfache Steigerung des katalytischen Stroms im direkten Vergleich zu einer Bi-Schicht $(n=1)$ beobachtet worden. Den Nachweis für einen effizienten Elektronentransfer innerhalb der Architektur liefern hierbei die offensichtliche Zunahme des katalytischen Stroms sowie die Menge an elektroaktivem Cyt $c$ mit zunehmender Zahl an abgeschiedenen Bi-Protein-Schichten. Die erhaltenen Daten implizieren auch, dass die $\mathrm{CDH}$ - und Cyt $c$-Moleküle in den verschiedenen Proteinstapeln innerhalb der Multischichtanordnung in elektrischem Kontakt mit der Elektrode stehen. Infolge der Laktosediffusion in das Multischichtsystem hinein, führt dies zu einer kaskadenartigen Erhöhung der Umsatzaktivität.

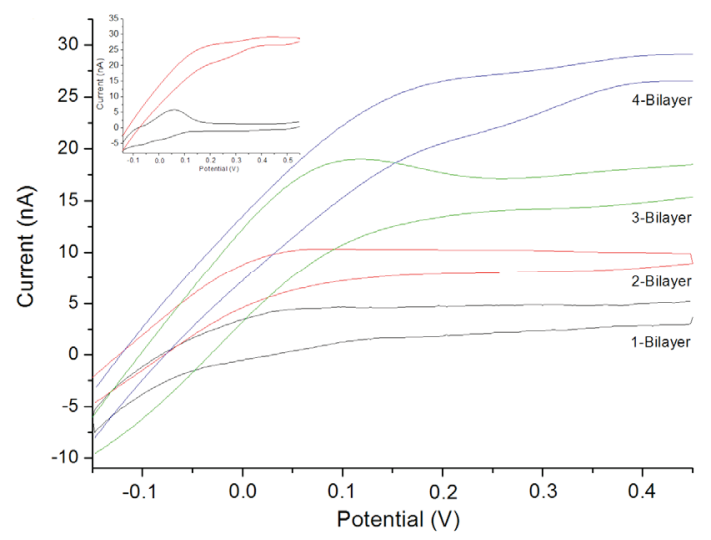

Fig. 4. Zyklovoltammogramme von verschiedenen Au-MUA/MU-cyt c-[SiNP-CDH॰cyt c]n supramolekularen Elektroden mit unterschiedlicher Zahl an immobilisierten Schichten. Katalytischer Strom der CDH-basierten Multischichtelektroden $(n=1,2,3,4)$ in Anwesenheit von Laktose (5 mM) bei einer Scanrate von $5 \mathrm{mV} / \mathrm{s}$. Inset: Zeigt eine 4-Bi-Schichtelektrode mit und ohne Laktose.

Basierend auf diesem modularen Prinzip wurde auch die Laccase (Lac) für den Sauerstoffnachweis in multiple Schichten eingebettet. Auch in diesem Fall machten wir uns die unterschiedlichen Ladungen der Proteine zu Nutze. $\mathrm{Da}$ die Lac bei pH 7 eine negative Nettoladung aufweist und Cyt $c$ eine positive, war es möglich diese für die Bildung der Präkomplexe bestehend aus Cyt $c$ und Lac in einer definierten Mischung zu nutzen (Abb. 5). Die katalytische 
Aktivität der Laccase wurde bei negativeren Potentialen untersucht. In den CV-Experimenten kann ein signifikanter Reduktionsstrom aufgrund der Sauerstoffreduktion durch die Lac beobachtet werden (Abb. 6).

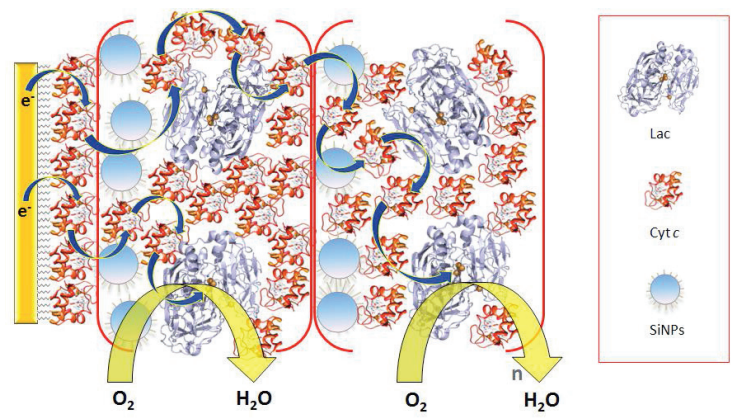

Elektronentrasnferschritte des katalytischen Prozesses innerhalb der Multischichtarchitektur:

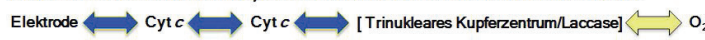

Fig. 5. Schematische Darstellung der Struktur und der postulierten Elektronentransferkaskade einer AuMUA/MU-Cyt c-[SiNPs/Cyt c.Lac]-Multischichtelektrode. Die Cyt c-Monoschicht wurde auf einer gemischten Thiolschicht (MUA/MU) assembliert. Die multiplen Schichten bestehen aus [SiNPs/Cyt c $\cdot$ Lac]n ( $n=1,2$, 3, 4, 5, 6 Bi-Schichten).

Der katalytische Strom resultiert aus der Reduktion von Cyt $c$, welcher durch die nachfolgende Oxidation von Cyt $c$ mittels benachbarter Laccasemoleküle noch verstärkt wird. Die erhaltene Sauerstoffreduktion im Potentialbereich der Cyt $c$-Umwandlung verifiziert die postulierte Funktionalität der Proteinarchitektur. Somit kann auch für diese System eine Signalkette postuliert werden: Diese geht von der Elektrode aus, an welcher Cyt $c$ reduziert wird und die Elektronen anschließend infolge von Cyt $c$-Cyt $c$ Interproteinelektronentransfer auf das T1-Zentrum der Laccase und von dort auf das dreikernige Kupferzentrum übertragen werden, an welchem die Elektronen dann schließlich für die Reduktion von molekularem Sauerstoff zu Wasser genutzt werden (Abb. 5). Um den Einfluss der Zahl an immobilisierten Laccaseschichten auf den katalytischen $\mathrm{Re}$ duktionsstrom zu beurteilen, wurde der katalytische Strom bei $-200 \mathrm{mV}$ gegen $\mathrm{Ag} / \mathrm{AgCl}$ in luftgesättigtem Puffer für Elektroden mit unterschiedlicher Schichtzahl bestimmt (Abb. 6).

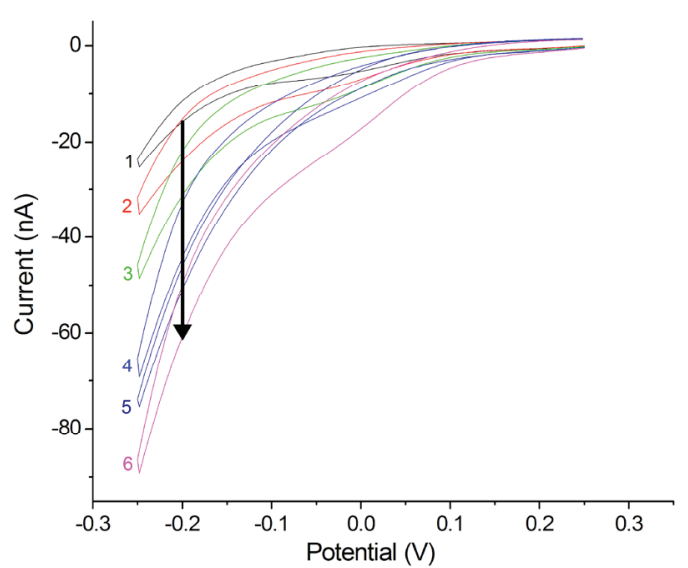

Fig. 6. Zyklovoltammogramme von Multischichtelektroden Au-MUA/MU-Cyt c-[SiNPs/cyt c.Lac] $]_{1-6}$ mit unterschiedlicher Schichtzahl in luftgesättigtem Puffer (Scanrate $3 \mathrm{mV} / \mathrm{s}, 20 \mathrm{mM}$ Phosphate-Citrat Puffer pH 4.5). Die katalytischen Reduktionsströme wurden bei einem Potential von $-200 \mathrm{mV}$ vs $\mathrm{Ag} / \mathrm{AgCl}$ ausgewertet. 1-6 gibt die Anzahl der Bi-Schichten auf der Elektrode wieder.

Aus den Zyklovoltammogrammen kann geschlossen werden, dass der Reduktionsstrom mit der Zahl an Bi-Schichten ansteigt. Dies legt wiederum nahe, dass infolge der zunehmenden Zahl an katalytischen Zentren (Menge an Laccase) auch die bioelektrokatalytische Umwandlung von Sauerstoff gesteigert wird. Die Schlussfolgerung wird zusätzlich durch die Zunahme der Menge des als Elektronen-„,Shuttle“ wirkenden, elektroaktiven Cyt $c$ unterstützt.

Basierend auf der nachgewiesenen Funktionalität der beiden enzymatischen Multischichtsysteme bestehend aus $\mathrm{CDH} / \mathrm{Cyt} c$ bzw. Lac/Cyt $c$ und inrer unterschiedlichen Potentialbereiche sollten diese in einem fortgeschrittenen System gemeinsam in nur einer Multischichtarchitektur vereinigt werden. Dadurch soll eine Schaltung bzw. ein Aktivitätswechsel zwischen zwei co-immobilisierten Enzymen innerhalb eines schichtweise aufgebauten supramolekularen Netzwerks ermöglicht werden. Die Kontrolle der Aktivität der Enzyme innerhalb des Systems soll durch das angelegte Potential erzielt werden (Abb. 7). 


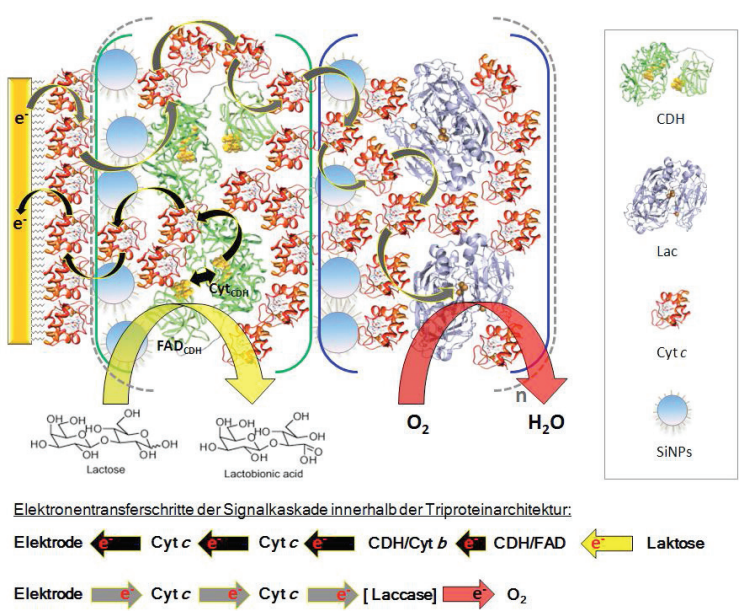

Abb. 7. Darstellung einer [SiNPs/CDH・Cyt c/SiNPs/ Lac.Cyt c $]_{n}$ Multiproteinarchitektur, assembliert auf einer Cyt c-Monoschichtelektrode. Die Cyt c Monoschicht wurde auf einer gemischten Thiolschicht (MU/MUA) adsorbiert. Schichtstruktur $(n=1,2,3,4$ Tetraschichten).

Die Wechselwirkung der beiden Enzyme (CDH und Lac) mit Cyt $c$ wurde anhand der beiden bisher diskutierten Systeme schon nachgewiesen. Die weiterführende duale Enzymarchitektur ist als schaltbares System für den dualen Nachweis zweier unterschiedlicher Analyte, Laktose und Sauerstoff konzipiert. In diesem Fall fixierten wir die $\mathrm{CDH}-\mathrm{Cyt} c$ - und Lac-Cyt $c$-Interproteinelektronentransferreaktion auf der Elektrodenoberfläche und koppelten sie sowohl mit Cyt $c$-Cyt $c$ Selbstaustauschreaktionen als auch mit dem direkten Elektrontransfer der Cyt $c$-Moleküle mit der Elektrode. Die Funktionalität dieser komplexen Triproteinarchitektur wurde mittels CV untersucht. Hierbei konnte gezeigt werden, dass die Triproteinelektrode weiterhin für den selektiven Laktosenachweis $(C D H)$ genutzt werden kann und die katalytische Aktivität der Elektrode auch weiterhin von der Anzahl immobilisierter Schichten abhängig ist (Abb. 8, Tab. 1). Hervorgehoben sei hier, dass eine unterschiedliche Anzahl von Tetraschichten (SiNPs/CDH・Cyt $c$, SiNPs/Lac.Cyt $c$ ) eingesetzt wurde.

Zusätzlich ist ersichtlich, dass die Anwesenheit eines weiteren Biokatalysators in Form der Laccase sich nicht negativ auf die Aktivität der $\mathrm{CDH}$ auswirkt. In gleicher Weise wurde die Triproteinelektrode auch bezüglich ihrer biokatalytischen Eigenschaften gegenüber Sauerstoff (Lac) untersucht. Auch hier kann gezeigt werden, dass die Umsetzung von Sauerstoff durch die Laccase weiterhin ohne Störung möglich ist. Zudem besteht auch für die biokatalytische Aktivität gegenüber Sauerstoff weiterhin eine Abhängigkeit von der Zahl an immobilisierten Schichten und somit der Menge an Biokatalysator welcher in die Schichtarchitektur eingebettet wurde (Abb. 9, Tab. 1).

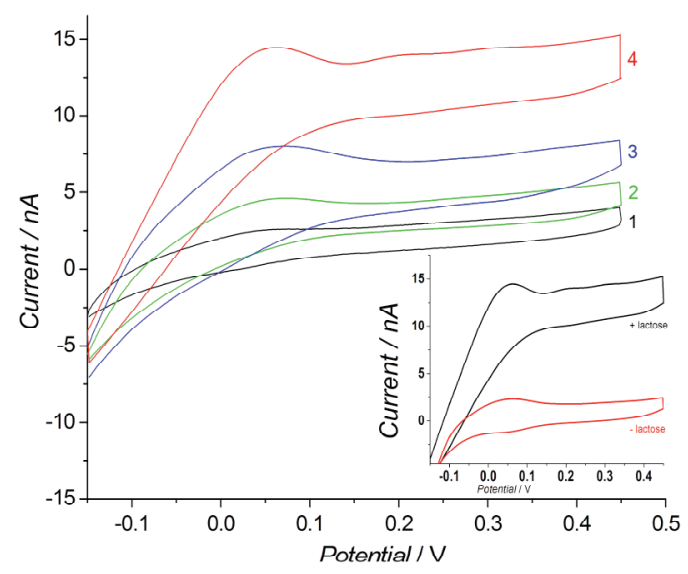

Fig. 8. Zyklovoltammogramme von Au-MUA/MUCyt c-[SiNPs/Cyt c.CDH/SiNPs/Cyt c $\cdot$ Lac $]_{1-4} \quad$ Elektroden (Tetraschichten $(n=1,2,3,4)$ in Anwesenheit von Laktose $5 \mathrm{mM}$ (Scanrate $3 \mathrm{mV} / \mathrm{s}, \mathrm{pH}$ 4.5, $20 \mathrm{mM}$ Phosphat-Citrat Puffer). Inset: Zyklovoltammogramm einer 4-Tetraschichtelektrode in An- und Abwesenheit von Laktose.

Mit dieser supramolekularen Architektur ist daher gelungen, eine bidirektionale Elektrontransferkaskade für den dualen Substratnachweis zu etablieren. Um die Laccase in einen aktiven Zustand zu überführen, ist es notwendig Elektronen für die Sauerstoffreduktion bereitzustellen. Für eine Aktivierung der $\mathrm{CDH}$ wiederum müssen die während der Zuckeroxidation freigesetzten Elektronen gesammelt werden. Cyt $c$ übernimmt in diesem System die zentrale Aufgabe eines Elektronendonors/ Elektronenakzeptors. Durch Justierung des Redoxzustands von Cyt $c$ ist es im gesamten supramolekularen Netzwerk möglich, die Aktivität der beiden Biokatalysatoren einfach an- und auszuschalten. Damit folgt dieses System nicht nur Anforderungen aus der Analytik sondern nutzt Prinzipien biologischer Signalwege und transferiert sie auf das Gebiet neuer biohybrider Funktionssysteme.

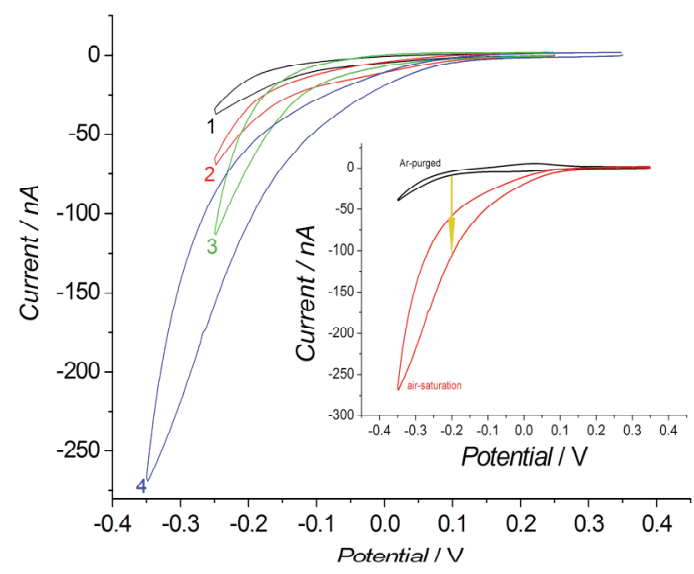

Abb. 9. Zyklovoltammogramme von Au-MUA/MUCyt c-[SiNP/Cyt c.CDH/SiNPs/Cyt c $\cdot{ }^{2}$ Lac $]_{1-4}$ Elektroden mit unterschiedlicher Schichtzahl; vermessen in luftgesättigtem Puffer (Scanrate $3 \mathrm{mV} / \mathrm{s}, \mathrm{pH}$ 4.5, 20 mM Phosphat-Citrat Puffer). Inset: Zyklovoltam- 
mogramm einer 4-Tetraschicht-Elektrode in luftgesättigtem und Argon-gespülten Puffer.

Tab. 1: Katalytische Ströme und Cyt c-Konzentrationen der Triproteinarchitekturen mit 1-4 Tetraschichten.

\begin{tabular}{|c|c|c|c|c|}
\hline $\begin{array}{c}\text { Dual } \\
\text { Enzyme } \\
\text { Elektroden }\end{array}$ & $\begin{array}{c}\text { Anzahl } \\
\text { Tetra- } \\
\text { Schichten }\end{array}$ & $\begin{array}{c}\text { Cyt } c \\
\text { Konz. }^{[\mathrm{a}]} \\
\mathrm{pmol} / \mathrm{cm}^{2}\end{array}$ & $\begin{array}{c}\text { Kat. } \\
\text { Strom }{ }^{[b]} \\
\operatorname{Lac}(n A)^{[c]}\end{array}$ & $\begin{array}{c}\text { Kat. } \\
\text { Strom }^{[\mathrm{b}]} \\
\mathrm{CDH} \\
(\mathrm{nA})^{[\mathrm{d}]}\end{array}$ \\
\hline $\mathrm{CDH} \cdot \mathrm{Lac}$ & 1 & $21 \pm 2$ & $-26 \pm 1$ & $4 \pm 1$ \\
\hline $\mathrm{CDH} \cdot \mathrm{Lac}$ & 2 & $40 \pm 3$ & $-45 \pm 2$ & $6 \pm 1$ \\
\hline $\mathrm{CDH} \cdot \mathrm{Lac}$ & 3 & $63 \pm 3$ & $-70 \pm 6$ & $9 \pm 2$ \\
\hline $\mathrm{CDH} \cdot \mathrm{Lac}$ & 4 & $79 \pm 8$ & $-110 \pm 11$ & $15 \pm 2$ \\
\hline
\end{tabular}

[a] Die Cyt c-Konzentrationen wurden anhand der Peakfläche im CV bei einer Scanrate von $100 \mathrm{mV} / \mathrm{s}$ berechnet. [b] Die katalytischen Ströme wurden nach Zugabe von $5 \mathrm{~mm}$ Laktose (CDH, bei positiver Polarisation) oder in luftgesättigtem Puffer (Lac, bei negativer Polarisation) bei einer Scanrate von 3 $\mathrm{mV} / \mathrm{s}$ bestimmt. [c] Bei $-200 \mathrm{mV}$. [d] Bei $+450 \mathrm{mV}$.

Um den Einfluss der Substratkonzentration auf die proteinbasierte Signalketten zu untersuchen, wurden die Proteinelektroden bei verschiedenen Laktose- (für die $\mathrm{CDH}$ ) und Sauerstoffkonzentrationen (für die Lac) analysiert. Die Daten zeigen sowohl eine lineare Abhängigkeit des Reduktionsstroms von der $\mathrm{O}_{2}$-Konzentration (0-100\% Luftsättigung) als auch eine nahezu lineare Abhängigkeit des Oxidationsstroms bezogen auf den Laktosegehalt in Lösung (0.02-1.0 mM). Somit ist das duale Enzymsystem in der Lage, den erhöhten Sauerstoff- und Laktosekonzentrationen zu folgen. Diese Beobachtung legt nahe, dass der Gesamtstrom innerhalb der Triproteinarchitektur zumindest im untersuchten Konzentrationsbereich durch die katalytische Reduktion (Lac) oder Oxidation (CDH) an den Enzymen begrenzt wird und nicht durch die Reaktionen zwischen den Enzymen und Cyt $c$, Cyt $c$ und Cyt $c$ oder zwischen Cyt $c$ und der Elektrode.

\section{Zusammenfassung}

Es wurden unterschiedliche Multischicht-basierte Redoxprotein-Enzymelektroden für den selektiven und sensitiven Substratnachweis aufgebaut. In einem weiterentwickelten System konnte sogar eine nanobiomolekulare Proteinarchitektur mit zwei funktionalen Signalketten auf der Basis von Protein-Protein-Wechselwirkungen auf Elektroden realisiert werden. Hierzu wurden die $\mathrm{CDH}$ und die Lac, welche zuvor einzeln in die Cyt $c$-basierten Multischichtarchitekturen integriert waren, in nur einer Architektur vereinigt. Innerhalb dieses Triproteinsystems sind die beiden Enzyme über Cytochrom $c$ in einem immobilisierten Zustand effizient mit der Elektrode kontaktiert. Da die Aktivität der Enzyme durch die Bereitstellung bzw. den Entzug der Elektronen kontrolliert wird, kann der Redoxzustand von Cyt $c$ dafür genutzt werden, die Aktivität der beiden Bio- katalysatoren an- und auszuschalten. Desweiteren arbeiten die schaltbaren Reaktionskaskaden der Lac und $\mathrm{CDH}$ in einer unseparierten Matrix, ohne dass die Anwesenheit des anderen Biokatalysators stört. Innerhalb des modularen Multischichtsystems agiert Cyt $c$ auf Basis von intermolekularem Elektronentransfer als bi-direktionaler molekularer Draht. Es ist zu erwarten, dass dieser Ansatz einen Beitrag für die Weiterentwicklung von MultiplexProteinelektroden und Biosensoren leistet.

\section{References}

[1] S.V. Dzyadevych, V.N. Arkhypova, A.P. Soldatkin, A.V. Elskaya, C. Martelet, N. JaffrezicRenault, Amperometric enzyme biosensors: past, present and future. IRBM 29, 171 (2008).

[2] J.F. Cassidy, A.P. Doherty, J.G. Vos, (1998) Principles of chemical and biological sensors. Wiley, Toronto (1998).

[3] A. Ramanavicius, A. Ramanaviciene, A, Malinauskas, Electrochim. Acta 51, 6025, (2006).

[4] G. Decher, Science 277, 1232 (1997).

[5] T. Ikeda, Electrochemical biosensors based on biocatalyst electrodes. Bull. Electrochem. 8, 145-159, (1992).

[6] Willner, I.; Willner, B.; Katz, E. Biomoleculenanoparticle hybridsystems for bioelectronic applications. Bioelectrochemistry, 70, 2-11 (2007).

[7] E. Katz, A.N. Shipway, I. Willner, In Biochemical Fuel Cells, Vielstich, W., Gasteiger, H. A., Lamm, A., Eds.; Wiley and Sons: London, Vol. 1, pp 1-27, (2003).

[8] S. Borgmann, G. Hartwich, A. Schulte, W. Schuhmann, In Electrochemistry of Nucleic Acids and Proteins. Towards Electrochemical Sensors for Genomics and Proteomics; E: Palecek, F. Scheller, J. Wang, Eds.; Perspectives in Bioanalysis; Elsevier: Amsterdam, Vol. 1, Chapter 17, pp 599-655 (2005).

[9] C. Léger, P. Bertrand, Direct electrochemistry of redox enzymes as a tool for mechanistic studies. Chem. Rev. 108, 2379-2438 (2008).

[10] P. He, N. Hu, Electrocatalytic properties of heme proteins in layer-by-layer films assembled with $\mathrm{SiO}_{2}$ nanoparticles, Elelctroanalysis 16:11221131 (2003).

[11] H. Yang, Y. Zhu, A High Performance Glucose Biosensor Enhanced via Nanosized $\mathrm{SiO}_{2}$. Analytica Chimica Acta, 554, 92-97 (2005).

[12] Y. Sun, F. Yan, W. Yang, C. Sun, Multilayered construction of glucose oxidase and silica nanoparticles on Au electrodes based on layerby-layer covalent attachment. Biomaterials 27, 4042-4049 (2006). 